\title{
Stroking me softly: Body-related effects in effect-based action control
}

\author{
Robert Wirth $^{1} \cdot$ Roland Pfister $^{1} \cdot$ Janina Brandes ${ }^{2} \cdot$ Wilfried Kunde $^{1}$
}

Published online: 8 June 2016

(C) The Psychonomic Society, Inc. 2016

\begin{abstract}
Empirical investigations of ideomotor effect anticipations have mainly focused on action effects in the environment. By contrast, action effects that apply to the agent's body have rarely been put to the test in corresponding experimental paradigms. We present a series of experiments using the response-effect compatibility paradigm, in which we studied the impacts of to-be-produced tactile action effects on action selection, initiation, and execution. The results showed a robust and reliable impact if these tactile action effects were rendered task-relevant (Exp. 1), but not when they were task-irrelevant (Exps. 2a and 2b). We further showed that anticipations of tactile action effects follow the same time course as anticipations of environment-related effects (Exps. 3 and 4). These findings demonstrate that body-related action effects affect action control much as environment-related effects do, and therefore support the theoretical assumption of the functional equivalence of all types of action effects.
\end{abstract}

Keywords Action control · Effect anticipations .

Body-related feedback $\cdot$ Ideomotor theory

The question of how abstract movement intentions can be translated into observable motor behavior has occupied researchers for a long time. Ideomotor theory suggests a parsimonious solution to this question: According to this view, motor patterns are activated by recollecting the perceptual

Robert Wirth

robert.wirth@uni-wuerzburg.de

1 Department of Psychology, Julius Maximilians University of Würzburg, Würzburg, Germany

2 Neuropsychological Department, University of Hamburg, Hamburg, Germany changes that these patterns produce. For example, anticipating the darkness that comes with closing one's eyes is assumed to activate the very action of closing the eyelids. Early formulations of this theory emphasized body-related, and especially proprioceptive, action effects as a means to address the corresponding motor patterns (Harleß, 1861; Herbart, 1825; James, 1890; cf. Pfister \& Janczyk, 2012; Stock \& Stock, 2004). In other words, the mental representations of upcoming proprioceptive changes are assumed to be linked to the specific actions that had caused them, and by this linkage, sensory anticipations gain the potential to activate the corresponding actions.

The focus of these early theoretical formulations on bodyrelated action effects becomes evident in different writings and can be succinctly summarized in the following definition of "movement ideas" (i.e., anticipations) as "the revival, through central excitation, of the sensations, visual, tactile, kinaesthetic, originally produced by the performance of the movement itself" (Washburn, 1908, p. 280; see also Stock \& Stock, 2004). In contrast to this focus on body-related action effects, empirical investigations of the ideomotor mechanism have mainly focused on action effects in the environment that are external to the body (e.g., Greenwald, 1970; Hommel, 1993; Kunde, 2001). Typical experimental setups employ keypress actions that are contingently linked to either visual or auditory action effects generated by a computer. The repeated experience of this linkage results in a bidirectional association between the keypress and the following action effect. This association becomes evident when, later on, experiencing these effects induces the action (Elsner \& Hommel, 2001; Hoffmann, Lenhard, Sebald, \& Pfister, 2009; Wolfensteller $\&$ Ruge, 2011). Moreover, the anticipation of acquired action effects can be studied, given appropriate settings: When actions predictably produce a visual or auditory effect that mismatches this action on a shared dimension (e.g., a spatial left- 
right dimension), action production is delayed relative to conditions in which the action and effect match (Ansorge, 2002; Janczyk, Pfister, Crognale, \& Kunde,2012; Pfister, Kiesel, \& Melcher, 2010; Pfister \& Kunde, 2013; Rieger, 2007). The commonly held explanation of this response-effect (R-E) compatibility effect is that the codes of the experimentally manipulated auditory or visual effects interfere with the codes of the body-related effects, which become activated at some point in action generation (Hommel, Müsseler, Aschersleben, \& Prinz, 2001).

In contrast to the growing body of literature on such environment-related effects, the processing of body-related action effects has rarely been subject to empirical study. So far, only one study in the R-E compatibility paradigm has employed vibrotactile action effects to assess their impact on response production (Pfister, Janczyk, Gressmann, Fournier, \& Kunde, 2014). Participants were to press a left or a right key, and each response made one of the keys vibrate. The vibration occurred either at the key that had just been pressed (compatible condition) or at the opposite key (incompatible condition). The results yielded faster response times (RTs) for the compatible than for the incompatible condition. Because the vibration only started after the key was pressed, these findings indicate that the vibration effects were indeed anticipated during action planning and modulated response production, even though the vibration effects were presented after the response had been completed. This observation has provided first evidence that body-related action effects are also anticipated during action planning and can serve to access an agent's response repertoire.

Still, the effects found in this initial study on the role of bodyrelated action effects for action production (Pfister, Janczyk, Gressmann, et al., 2014) were small at best, which might come as a surprise, given that previous studies on the perception of body-related effects have reported highly systematic effects. This becomes apparent when considering work on the sensory attenuation of self-produced events, resulting in phenomena such as the inability to tickle oneself (Blakemore, Wolpert, \& Frith, 2000; Weiskrantz, Elliott, \& Darlington, 1971). Studies on sensory attenuation for body-related effects have assessed the perception of tactile stimulation that was produced either by the participants themselves or by the experimenter. Robust differences between these two conditions are typically interpreted as reflecting the operation of a forward model that allows predictions of upcoming stimulation for self-performed actions, but not for the actions of another agent (cf. Blakemore, Frith, \& Wolpert, 1999; Blakemore, Wolpert, \& Frith, 1998).

The tactile effects used in studies on sensory attenuation (Blakemore et al., 1998) arguably are quite different from the vibration effects that have been used to study effect anticipations in the R-E compatibility paradigm (Pfister, Janczyk, Gressmann, et al., 2014). Indeed, the mere observation of someone else being touched (akin to the effects used in studies on sensory attenuation) already generates activation in the somatosensory cortex that simulates this touch experience, suggesting that tactile stimuli are processed automatically and efficiently (Keysers et al., 2004). Therefore, the first aim of the present study was to establish an R-E compatibility paradigm that would produce robust indices of ideomotor effect anticipations relating to tactile action effects (Exps. 1 and 2).

A further apparent difference between the studies on sensory attenuation and on the anticipation of vibration effects is whether the tactile effects were task-relevant: Whereas the vibrotactile effects in the former studies could be ignored completely during task performance, in the latter the tickling sensation was the only salient event, and it even had to be rated afterward at times, rendering it task-relevant. To assess the impact of relevance on similar tactile action effects in an R-E compatibility paradigm, we designed Experiment 1 to include task-relevant tactile action effects, whereas in Experiments $2 \mathrm{a}$ and $2 \mathrm{~b}$ these effects were task-irrelevant (for a related approach using visual action effects, see Ansorge, 2002). Experiments $2 \mathrm{a}$ and $2 \mathrm{~b}$ further differed regarding visual feedback of the moving hand, with full feedback being available in Experiment 2a, and no feedback in Experiment $2 b$.

Furthermore, substantial evidence from different areas in psychology suggests that most cognitive operations affect not only the duration of action planning, but also how the planned action is executed (e.g., Buetti, Juan, Rinck, \& Kerzel, 2012; Dale \& Duran, 2011; Freeman \& Ambady, 2014; Pfister, Wirth, Schwarz, Steinhauser, \& Kunde, 2016; Song \& Nakayama, 2009; Spivey, Grosjean, \& Knoblich, 2005; Wirth, Pfister, Foerster, Huestegge, \& Kunde, in press; Wirth, Pfister, \& Kunde, 2016). Ideomotor theory does not draw a sharp distinction between action planning and execution; therefore, it is possible to expect an influence of anticipated action effects not only on measures that relate exclusively to action planning and initiation (such as RTs), but also on measures that relate to response execution. Moreover, it is likely that the planning of subsequent components of a continuous motor action takes place concurrently with the execution of one part of that action. Consequently, delays in action planning that affect the RT might also affect the duration of action execution (e.g., Adam et al., 2000). Corresponding findings have recently been reported for the anticipation of environment-related action effects (Pfister, Janczyk, Wirth, Dignath, \& Kunde, 2014; Wirth, Pfister, Janczyk, \& Kunde, 2015). Here, participants had to move a mouse cursor on a screen to either the left or the right to produce an action effect that could either be compatible (on the side toward which the cursor was moved) or incompatible (opposite the cursor's movement). Compatibility between the response and the effect influenced not only the duration of response selection and initiation, but continued its influence during response production, in the forms of slowed responses that would later produce incompatible rather than compatible effects and of a strong spatial attraction toward the location where the action 
effect would later appear. Therefore, in Experiments 1 and 2, we tested whether such effects during movement execution would equally emerge for tactile, body-related effects.

In addition to demonstrating anticipations of body-related action effects, we aimed to study the temporal dynamics of such anticipations during action selection, initiation, and execution (Exps. 3 and 4). For environment-related effects, previous studies in the R-E compatibility paradigm have suggested prominent roles of effect anticipations during both, action selection and action initiation (Kunde, 2003; Kunde, Koch, \& Hoffmann, 2004; Shin \& Proctor, 2012). More precisely, these studies implemented a precue that informed about the to-beperformed action, which only had to be initiated after a variable cue-go interval (CGI). This design showed larger R-E compatibility effects at short than at long CGIs (indicating a role of effect anticipations in action selection), while still yielding reliable R-E compatibility effects even at long CGIs (indicating a role of effect anticipations in action initiation). In Experiments 3 and 4, we adopted this approach to compare the temporal dynamics of environment-related and body-related effects.

\section{Experiment 1}

In Experiment 1, we tested for anticipations of tactile action effects in an R-E compatibility paradigm. To this end, we constructed an apparatus that allowed us to manipulate the relation between a participant's movements and the resulting tactile effects on the agent's body, without altering the visual or auditory reafferences of the action (see Fig. 1). Participants operated a slider on top of the apparatus with their dominant hand, while their non-dominant hand and the corresponding forearm was inserted in the apparatus. Each of the participants' hand movements was continuously translated into a brush stroke on their forearm, and the movement directions of the hand and the brush

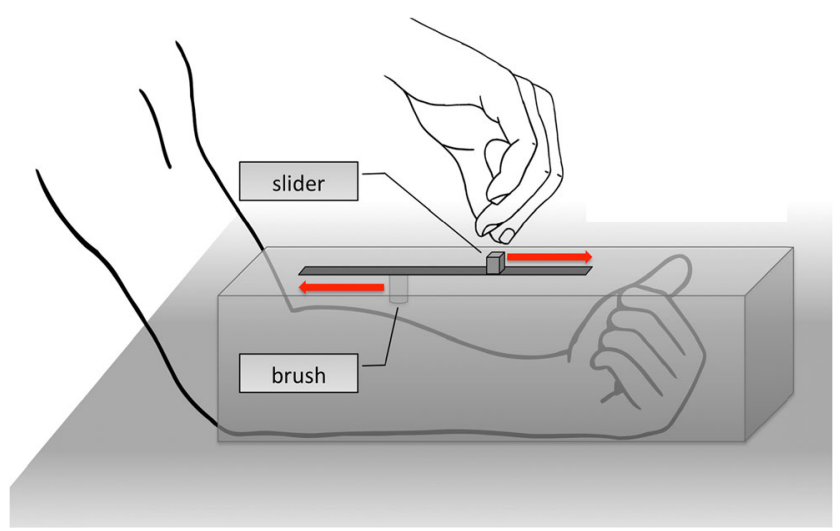

Fig. 1 Apparatus of the experiments. Participants operated a slider on top of the metal box with their dominant hand. The slider was connected to a brush that stimulated the arm of the non-dominant hand, and the brush moved either in the same direction as the slider (response-effect compatible condition) or opposite to the slider (response-effect incompatible condition). This figure depicts an incompatible trial either matched (compatible condition) or were opposite (incompatible condition) to each other. Participants were explicitly instructed to produce certain movements of the brush - that is, each target stimulus prompted them to move the brush either toward their hand or toward their arm crook - thereby rendering the tactile action effects task-relevant. R-E compatibility was manipulated trial to trial with target-inherent cueing (cf. Gaschler \& Nattkemper, 2012; Pfister, Janczyk, Wirth, et al., 2014; Pfister et al., 2010; Zwosta, Ruge, \& Wolfensteller, 2013).

For each response, we measured the initiation time (IT), movement time $(\mathrm{MT})$, and total RT $(\mathrm{RT}=\mathrm{IT}+\mathrm{MT})$, and expected longer latencies in the incompatible than in the compatible conditions for all three variables. Also, this effect of compatibility usually emerges especially in slow responses (since there is more time for the codes of the anticipated effect to interfere with the codes of the required response; Keller \& Koch, 2006; Kunde, 2001; Kunde, Lozo, \& Neumann, 2011; Pfister, Janczyk, Gressmann et al., 2014). We therefore expected to observe a larger influence of compatibility with slower than with fast responses, made evident by an interaction between R-E compatibility and distribution quintile.

\section{Method}

Participants Twenty-four participants were recruited (20 female, four male; two left-handed; mean age $=22.0$ years) and received monetary compensation. All participants reported normal vision and hearing and were naïve concerning the hypotheses of the experiment, and all provided written informed consent prior to the experiment.

Apparatus and stimuli The apparatus used in the experiment was a metal box with a slider on top (Fig. 1). The slider was moved with the dominant hand while the forearm of the nondominant hand rested in the metal box. The neutral position of the slider was at the center of the box, with a total movement distance to the final hand or crook position of $12 \mathrm{~cm}$ in both directions. The slider controlled a brush that stimulated the forearm in the box. The compatibility between the movement direction of the slider and the movement direction of the brush could be manipulated by means of pulses that were sent from the computer: In compatible trials, the brush moved in the same direction as the slider (e.g., the slider and the brush both moved toward the hand), whereas in incompatible trials, the brush moved in the opposite direction from the slider (e.g., the slider moved toward the hand, the brush moved toward the arm's crook). The brush moved at the same speed and to the same extent as the slider; only its direction was varied.

The stimuli consisted of two geometrical shapes (circle vs. square), each in two colors (green vs. blue). These shapes $(0.8$ $\mathrm{cm} \times 0.8 \mathrm{~cm}$ ) were presented centrally on the computer screen against a white background. The color informed participants of the location that the brush had to be moved to (hand vs. crook); 
the shape indicated the current compatibility relation between the slider and the brush (compatible vs. incompatible). The mapping of color and target direction, as well as the mapping of shape and compatibility relation, was counterbalanced across participants.

Procedure Participants were verbally instructed on the relevant mappings of color and shape and the trial-by-trial variation of the compatibility between the slider movement and the brush direction. Following these instructions, participants completed one practice block and nine experimental blocks of 20 trials. Each block was followed by a short break.

The trial procedure is illustrated in Fig. 2. At the beginning of a trial, the slider had to be moved to the center of the box while the outline of a fixation circle was displayed on the screen. During this movement, the brush was lifted up so that there was no stimulation on the arm. As soon as the center was reached, the computer program waited for a dwell time of 500 $\mathrm{ms}$, indicated by a filled fixation circle on screen. The counter restarted whenever the slider left the center position. After the dwell time, the target appeared on the screen, its color and shape varying randomly on a trial-by-trial basis. Simultaneously, the brush dropped on the forearm, so that from this point onward, a movement of the slider would provoke a tactile feedback. Now the brush had to be moved according to the target — either to the arm crook or to the hand - by pushing the slider in the corresponding direction, depending on the current compatibility condition. The target disappeared after $200 \mathrm{~ms}$ and left participants with a blank screen until the brush reached the crook or hand position. Auditory error feedback was provided if participants moved the brush to the wrong location. At the end of the trial, the brush was lifted again, so that the slider could be moved toward the center again without tactile stimulation.
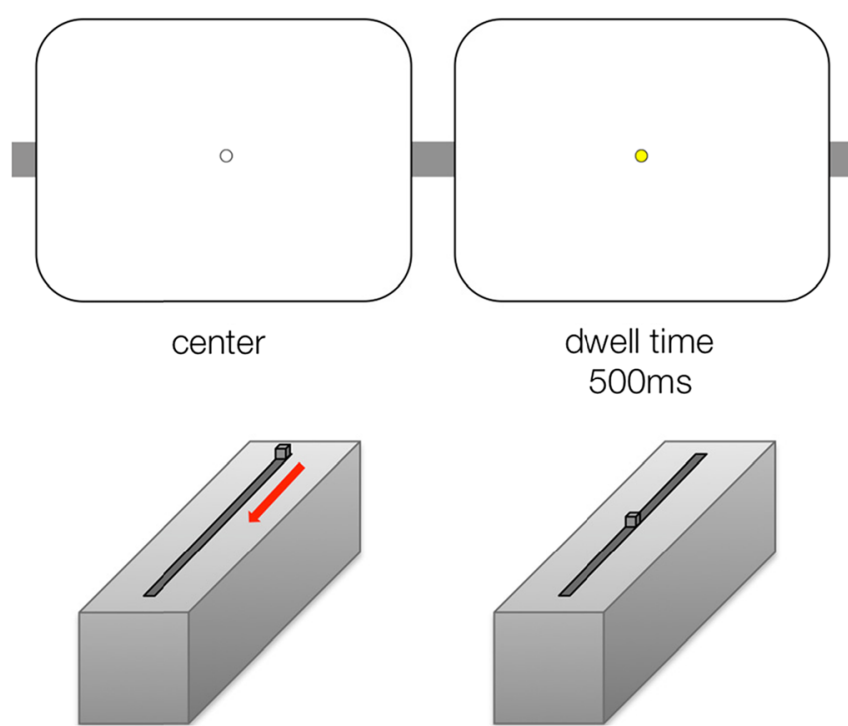

Fig. 2 Trial procedure. Participants started each trial by moving the slider to the center of the metal box. Upon reaching the center, the white fixation circle on the screen changed to yellow, indicating that participants had reached the center position. After a dwell time of $500 \mathrm{~ms}$, a colored shape
The IT was defined as the time from target onset to when the slider left the center position; the MT was measured from this point until the slider hit the hand or crook position. The RT was computed as the sum of IT and MT.

\section{Results}

For all following analyses, we omitted the practice block, as well as error trials (2.4\%); the error rates was $1.7 \%$ in compatible trials versus $3.0 \%$ in incompatible trials, $t(23)=1.93, p=.067, d=$ 0.54 . Equally, all trials with initial movements in the wrong direction were excluded ( $7.8 \%$ of all trials), which occurred equally often in both R-E compatibility conditions, $t(23)=1.09, p=.287$, $d=0.19$. Trials with ITs or MTs deviating more than $2.5 S D \mathrm{~s}$ from their cell mean were discarded as outliers, calculated separately for each participant and compatibility condition (4.2\%); $4.5 \%$ of the trials were removed in compatible trials, and $3.8 \%$ in incompatible trials, $t(23)=2.53, p=.019, d=0.59$.

To analyze the time course of the compatibility effect and allow for a direct comparison with previous analyses (Kunde, 2001; Pfister, Janczyk, Gressmann, et al., 2014), the three measures were then analyzed in separate $2 \times 5$ repeated measures analyses of variance (ANOVAs), with R-E Mapping (compatible vs. incompatible) and Distribution Quintile (1-5) as withinsubjects factors, and quintiles being computed separately for each dependent variable (see Fig. 3 for the corresponding means). For the sake of brevity, we do not report the main effects of distribution quintile, although they were, of course, significant across all experiments $(p \mathrm{~s}<.001)$.

Initiation time ITs were lower in the compatible condition (769 $\mathrm{ms})$ than in the incompatible condition $(989 \mathrm{~ms}), F(1$,
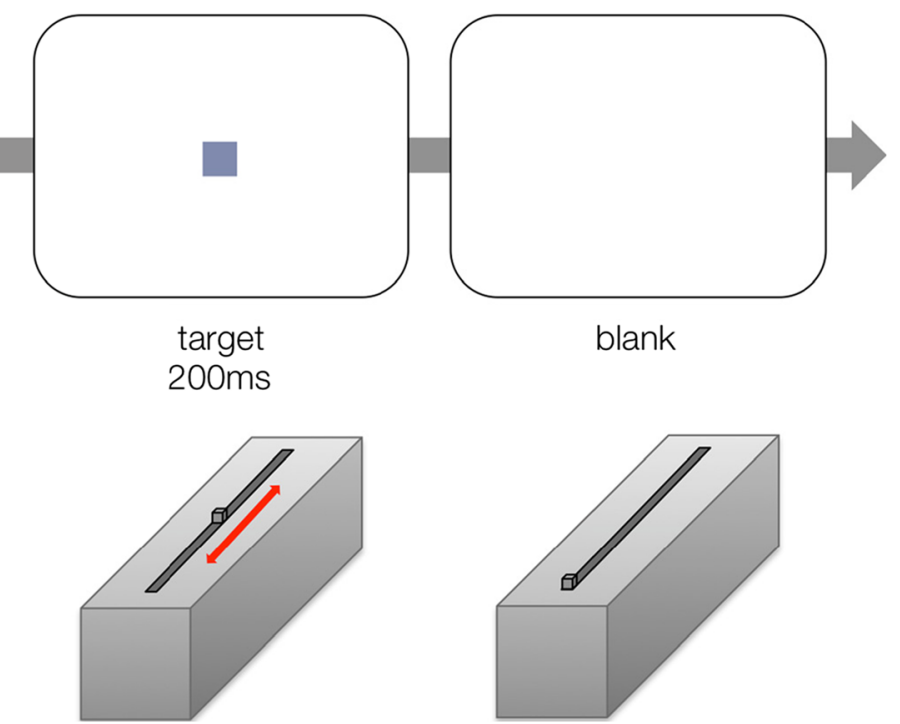

appeared as target, instructing participants about the correct movement direction of the brush and the current compatibility relation between the hand and brush movements 


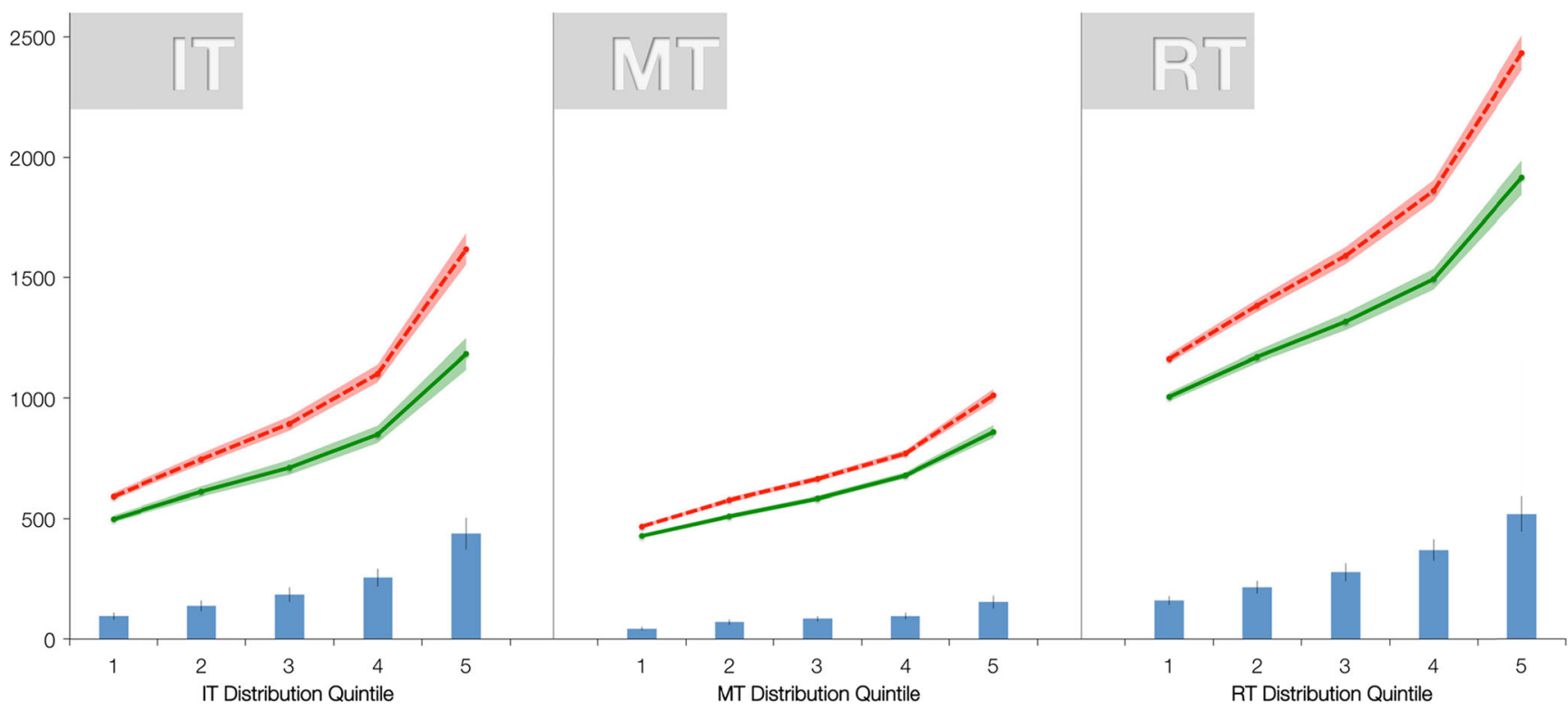

Fig. 3 Results of Experiment 1: Mean initiation times (IT, left panel), movement times (MT, center panel), and overall response times (RT $=$ IT + MT, right panel), in milliseconds, shown separately for their respective distribution quintiles (abscissa) and R-E mappings (continuous line $=$ compatible; dashed line = incompatible). Each value is depicted with the corresponding standard error of paired differences $\left(S E_{\mathrm{PD}}\right)$, calculated

separately for each quintile (Pfister \& Janczyk, 2013). Bars indicate the size of the compatibility effects per distribution quintile with their $S E_{\mathrm{M}} \mathrm{S}$. Please note that the quintile means of IT and MT do not sum to the corresponding RT quintile because the variables were binned independently

$23)=46.91, p<.001, \eta_{\mathrm{p}}{ }^{2}=.67$. A significant interaction between distribution quintile and R-E mapping, $F(4,20)=7.42, p$ $=.001, \eta_{\mathrm{p}}{ }^{2}=.60$, was driven by larger R-E compatibility effects for higher quintiles (see Fig. 3, left panel), even though the R-E compatibility effects was significant for each individual quintile, $t \mathrm{~s}>5.76, p \mathrm{~s}<.001$ (note that a Bonferroni-corrected alpha level would correspond to $\alpha / 5=.05 / 5=.01$ ).

Movement time Movements were faster in the compatible condition (609 ms) than in the incompatible condition (697 ms), $F(1$, $23)=41.72, p<.001, \eta_{\mathrm{p}}{ }^{2}=.65$. A significant interaction between distribution quintile and R-E mapping, $F(4,20)=5.24, p=.005$, $\eta_{\mathrm{p}}{ }^{2}=.51$, was driven by larger R-E compatibility effects for higher quintiles (see Fig. 3, center panel), though significant effects were present for all individual quintiles, $t \mathrm{~s}>5.37, p \mathrm{~s}<.001$.

Response time Responses were faster in the compatible condition (1379 $\mathrm{ms})$ than in the incompatible condition (1685 $\mathrm{ms}), F(1,23)=65.09, p<.001, \eta_{\mathrm{p}}{ }^{2}=.74$. The interaction between distribution quintile and R-E mapping was significant, $F(4,20)=9.71, p<.001, \eta_{\mathrm{p}}{ }^{2}=.66$, with larger R-E compatibility effects for higher quintiles (see Fig. 3, right panel). Reliable R-E compatibility effects again emerged for each individual quintile, $t \mathrm{~s}>6.93, p \mathrm{~s}<.001$.

\section{Discussion}

In Experiment 1, we conceptually replicated previous results (Pfister, Janczyk, Gressmann, et al., 2014) by showing that the anticipation of body-related action effects that are spatially incompatible with the corresponding movement direction interfere with the planning and/or initiation of this response (as indicated by R-E compatibility effects on ITs). The MT analysis further indicated that the compatibility manipulation also affected movement execution (for converging evidence, see Pfister, Janczyk, Wirth, et al., 2014; Wirth et al., 2015). The interaction between R-E compatibility and distribution quintile, which emerged for all measures, demonstrated that compatibility effects are especially strong for slow responses. This observation in consistent with the idea that anticipations need to be generated endogenously, so that they take some time to exert their full influence on action planning (Kunde, 2001).

These results provide strong evidence for the anticipation of tactile action effects during action planning. Importantly, however, the tactile action effects were explicitly task-relevant in Experiment 1. Task relevance is known to boost R-E compatibility effects (Ansorge, 2002; Janczyk, Yamaguchi, Proctor, \& Pfister, 2015), even though R-E compatibility effects based on the manipulation of task-irrelevant environment-related effects have been obtained, as well (e.g., Kunde, 2001). We therefore aimed at complementing the results by repeating Experiment 1 with task-irrelevant action effects.

\section{Experiment 2a}

To test the role of task relevance in the data obtained in Experiment 1, we replicated the previous setup, but now 
allocated less focus to the tactile feedback (i.e., the brush movement): Each target now prompted a specific slider direction instead of a brush direction. To allow for visual attention on the motor response, we further employed acoustical instead of visual stimuli. If tactile action effects are attended automatically, this setup should replicate the results of Experiment 1. If, however, tactile action effects need to be task-relevant to modulate response initiation and execution, the compatibility effects obtained in Experiment 1 should vanish.

\section{Method}

Participants A new set of 24 participants were recruited (18 female, six male; two left-handed; mean age $=27.8$ years) and received monetary compensation. All participants reported normal vision and hearing and were naive concerning the hypotheses of the experiment, and all provided written informed consent prior to the experiment. Five of the participants were excluded from the analyses due to high error rates $(>25 \%){ }^{1}$

Apparatus and stimuli The apparatus was identical to the one used in the Experiment 1. To allow participants to focus their attention on their hand and the response that they were required to perform, the stimuli were now presented acoustically. They consisted of either $400-\mathrm{Hz}$ or $800-\mathrm{Hz}$ tones (low vs. high) of 500-ms duration that were played on one of two MIDI instruments (marimba vs. dulcimer). The frequency of the tones informed participants of the location toward which the slider had to be moved (hand vs. crook), and the instrument indicated the current compatibility between the slider and the brush (compatible vs. incompatible). The mapping of tone frequency and slider direction, as well as the mapping of instrument and compatibility relation, was counterbalanced across participants.

Procedure The procedure of Experiment 2a was largely identical to that of Experiment 1, with the following modifications: Whether participants had reached the center position was now indicated by an LED that was mounted on the metal box. The light was turned on as soon as the slider was moved to the center position (akin to the yellow fixation circle in Exp. 1). After a corresponding dwell time had started, the target stimulus was presented via headphones, in the same way that the auditory error feedback was provided. Both tone frequency and instrument were randomized on a trial-by-trial basis. The instructions now focused on whether the slider had to be moved to the crook or the hand position, irrespective of the tactile feedback that this movement would provoke.

\footnotetext{
${ }^{1}$ Even when we included all 24 participants in the analysis, the results did not change for R-E mapping, $F \mathrm{~s}<0.51, p \mathrm{~s}>.481$, or the interaction of distribution quintile and R-E mapping, $F_{\mathrm{S}}<0.95, p \mathrm{~s}>.459$, for any of the variables.
}

\section{Results}

We again omitted the practice block, as well as all trials with errors (5.1\%), which occurred equally often in both R-E compatibility conditions, $t(18)=0.52, p=.606, d=0.13$. Likewise, all trials with initial movements in the wrong direction were excluded (3.2\% of all trials), which again occurred equally often in both R-E compatibility conditions, $t(18)=1.57, p=$ $.130, d=0.41$. Trials with ITs or MTs that deviated more than $2.5 \mathrm{SDs}$ from their cell mean were discarded as outliers, which were calculated separately for each participant and compatibility condition (3.6\%) and occurred equally often in both R-E compatibility conditions, $t(18)=0.53, p=.600, d=0.15$.

The three measures were then analyzed in separate $2 \times 5$ repeated measures ANOVAs with R-E Mapping (compatible vs. incompatible) and Distribution Quintile (1-5) as withinsubjects factors (see Fig. 4 for the corresponding means).

Initiation time Neither the main effect of R-E mapping, $F(1$, 18) $=0.90, p=.356, \eta_{\mathrm{p}}{ }^{2}=.05$, nor the interaction between distribution quintile and R-E mapping, $F(4,15)=0.52, p=$ $.726, \eta_{\mathrm{p}}{ }^{2}=.12$, reached significance (Fig. 4 , left panel).

Movement time As for ITs, we observed neither a main effect of R-E mapping, $F(1,18)=1.07, p=.315, \eta_{\mathrm{p}}{ }^{2}=.06$, nor an interaction of distribution quintile and R-E mapping, $F(4,15)$ $=1.18, p=.359, \eta_{\mathrm{p}}{ }^{2}=.24$ (Fig. 4, center panel).

Response time Again, neither the main effect of R-E mapping, $F(1,18)=0.29, p=.595, \eta_{\mathrm{p}}{ }^{2}=.02$, nor the interaction between distribution quintile and R-E mapping, $F(4,15)=$ $1.06, p=.409, \eta_{\mathrm{p}}{ }^{2}=.22$, reached significance (Fig. 4, right panel).

\section{Comparison to experiment 1}

To compare the sizes of the R-E compatibility effects in Experiments 1 and 2a, we computed a between-experiments analysis for each measure. These analyses were done by means of separate $2 \times 5 \times 2$ split-plot ANOVAs with R-E Mapping (compatible vs. incompatible) and Distribution Quintile (1-5) as within-subjects factors, and Experiment as a between-subjects factor.

To reduce redundancy, we focus on the interactions that included the factor Experiment; the critical interaction of RE mapping and experiment reached significance for all variables, $F_{\mathrm{S}}>28.21, p \mathrm{~s}<.001$. Equally, the three-way interaction was significant for all variables, $F_{\mathrm{S}}>4.13, p \mathrm{~s}<.007$.

\section{Discussion}

In Experiment 2a, we tested for an impact of task-irrelevant tactile action effects in the present R-E compatibility 


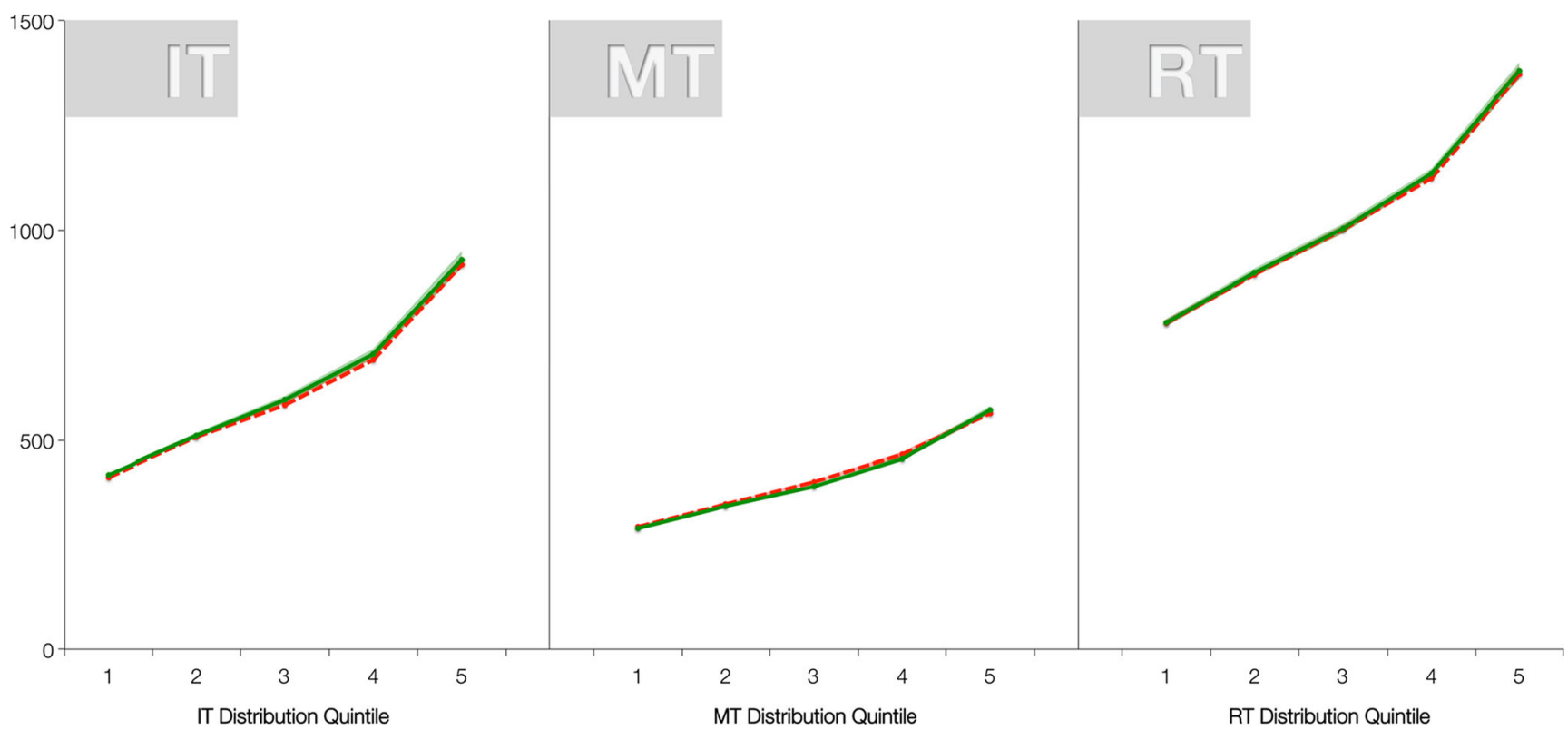
movement times (MT, center panel), and overall response times (RT, right panel), in milliseconds, shown separately for their respective distribution quintiles (abscissa) and R-E mappings (continuous line = compatible;
Fig. 4 Results of Experiment 2a: Mean initiation times (IT, left panel),

dashed line = incompatible). Each value is depicted with the corresponding standard error of the paired differences $\left(S E_{\mathrm{PD}}\right)$, calculated separately for each distribution quintile (Pfister \& Janczyk, 2013)

\section{Experiment 2b}

paradigm. Participants therefore were no longer asked to to move the slider, irrespective of the ensuing brush movement. In this setting, the tactile effects that the responses produced could be completely ignored. And indeed, whereas Experiment 1 had produced robust compatibility effects across all three measures, these compatibility effects completely vanished in Experiment 2a. Body-related action effects as studied in the present setup therefore only seem to occur during response selection if they constitute a discriminative and necessary feature of the required action.

In fact, the moving hand necessarily triggers effects other than the brush stroke that could similarly be used to represent and address the action. In addition to proprioceptive feedback, a feature that was likely to have been represented more strongly in Experiment 2a than in Experiment 1 was the visual representation of the moving hand, because this information allowed for controlling whether the intended end position of the slider had been reached. This difference might cause a dominance of the visual feedback of the moving hand, akin to other effects of visual dominance reported in the literature (Colavita, 1974; Colavita \& Weisberg, 1979; Posner, Nissen, \& Klein, 1976). In other words, the task-irrelevant bodily feedback might not have mattered (Kunde, Krauss, \& Weigelt, 2009; Kunde \& Weigelt, 2005; Mechsner, Kerzel, Knoblich, \& Prinz, 2001; Mechsner \& Knoblich, 2004) because the actions in Experiment 2a were represented by visuospatial features (see Janczyk, Skirde, Weigelt, \& Kunde, 2009, for converging evidence). This hypothesis will be addressed in Experiment 2b.
To test the hypothesis of visual dominance, we replicated Experiment 2a, but obstructed the participants' view of both the apparatus and their hand. A re-emerging R-E compatibility effect in this setting would indicate a critical role of visual dominance, whereas an absent R-E compatibility effect would suggest coding in terms of other features, such as proprioceptive feedback from the moving hand.

\section{Method}

Participants A new set of 24 participants were recruited (18 female, six male; two left-handed; mean age $=27.8$ years) and received monetary compensation. All participants reported normal vision and hearing and were naïve concerning the hypotheses of the experiment, and all provided written informed consent prior to the experiment. Two of the participants were excluded from the analyses due to high error rates $(>25 \%)^{2}$

Apparatus, stimuli, and procedure The experiment was mostly identical to Experiment $2 \mathrm{a}$. To obstruct the participants' view of their own hand and the apparatus, a cape was draped in front of them up to their shoulders. It was then

\footnotetext{
${ }^{2}$ Even when we included all 24 participants in the analysis, the results did not change for either the main effect of R-E mapping, $F \mathrm{~s}<0.91, p \mathrm{~s}\rangle$ .350 , or the interaction of distribution quintile and R-E mapping, $F \mathrm{~s}<$ $2.09, p s>.120$, for any of the variables.
} 
sufficiently tightened so that movements of the hand could not cause any wrinkles in the cape that could serve as a visual feedback of the action. Participants still moved the slider on the basis of a tone that was played via headphones, and the compatibility between the slider direction and the brush direction was still varied from trial to trial, but the sensation was not relevant for the completion of the task.

\section{Results}

We again omitted the practice block, as well as all trials with errors (4.1\%), which occurred equally often in both R-E compatibility conditions, $t(21)=0.98, p=.340, d=0.22$. Likewise, all trials with initial movements in the wrong direction were excluded ( $4.2 \%$ of all trials), which again occurred equally often in both R-E compatibility conditions, $t(21)=$ $1.19, p=.246, d=0.16$. Trials with ITs or MTs that deviated more than $2.5 S D$ s from their cell mean were discarded as outliers, which were calculated separately for each participant and compatibility condition (4.1\%) and occurred equally often in both R-E compatibility conditions, $t(21)=1.29, p=.211$, $d=0.40$.

The three measures were then analyzed in separate $2 \times 5$ repeated measures ANOVAs with R-E Mapping (compatible vs. incompatible) and Distribution Quintile (1-5) as withinsubjects factors (see Fig. 5 for the corresponding means).

Initiation time Neither the main effect of R-E mapping, $F(1$, 21) $=0.45, p=.835, \eta_{\mathrm{p}}{ }^{2}<.01$, nor the interaction between distribution quintile and R-E mapping, $F(4,18)=1.54, p=$ $.234, \eta_{\mathrm{p}}{ }^{2}=.26$, reached significance (Fig. 5, left panel).

Movement time As for ITs, we found neither a main effect of R-E mapping, $F(1,21)=0.66, p=.427, \eta_{\mathrm{p}}{ }^{2}=.03$, nor an interaction of distribution quintile and R-E mapping, $F(4,18)$ $=1.91, p=.152, \eta_{\mathrm{p}}{ }^{2}=.30$ (Fig. 5, center panel).

Response time Again, neither the main effect of R-E mapping, $F(1,21)=0.11, p=.743, \eta_{\mathrm{p}}{ }^{2}=.01$, nor the interaction between distribution quintile and R-E mapping, $F(4,21)=$ $1.30, p=.307, \eta_{\mathrm{p}}{ }^{2}=.22$, reached significance (Fig. 5, right panel).

\section{Discussion}

In Experiment 2b, we occluded the hands and the apparatus from the participants' view to test whether, without visual feedback, the R-E compatibility effects that we found in Experiment 1 would re-emerge. However, the compatibility of the slider direction and the brush direction did not influence performance. Visual dominance consequently does not seem to overwrite the tactile action effects, but they are generally not attended to if they are not a necessary and discriminatory feature of the task. Although task-relevant tactile action effects seem to be considered in action selection, initiation, and execution (Exp. 1), task-irrelevant action effects seem to be less important and are outweighed by other, task-relevant sensory

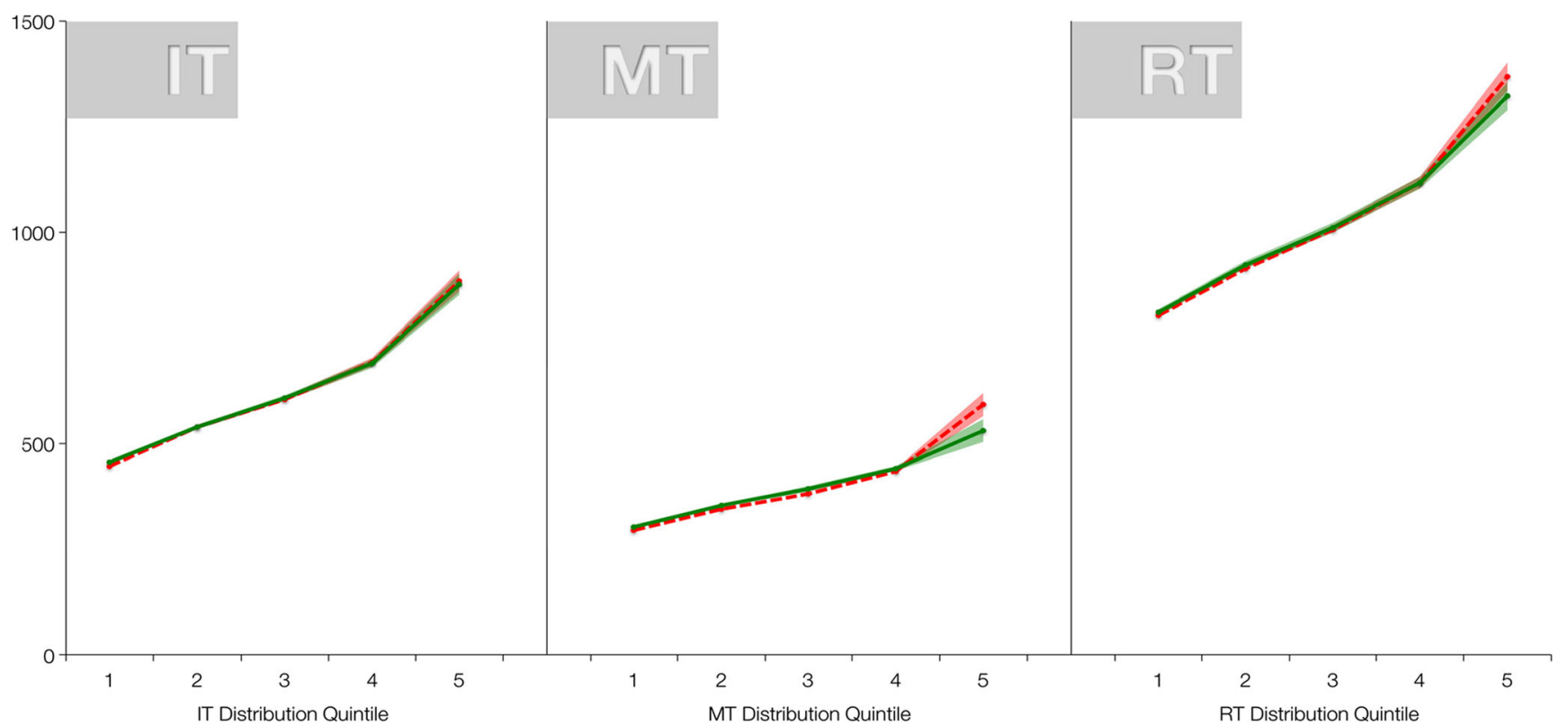

Fig. 5 Results of Experiment 2b: Mean initiation times (IT, left panel), movement times (MT, center panel), and overall response times (RT, right panel), in milliseconds, shown separately for their respective distribution quintiles (abscissa) and R-E mappings (continuous line = compatible;

dashed line = incompatible). Each value is depicted with the corresponding standard error of the paired differences $\left(S E_{\mathrm{PD}}\right)$, calculated separately for each distribution quintile (Pfister \& Janczyk, 2013) 
changes (e.g., the proprioceptive feedback of the moving hand).

An alternative view on this pattern of results would be to conceptualize the design of Experiment 1 as involving a tool transformation, whereas the designs of Experiments $2 \mathrm{a}$ and $2 \mathrm{~b}$ do not involve such a transformation. The present apparatus does, indeed, share several features with mechanical tools, with the incompatible R-E relation being similar to a firstclass lever with one pivot, in which hand movements in a certain direction (say, left) are continuously translated into brush movements to the opposite side (say, right). The difficulty associated with the R-E incompatible condition could thus be seen as a difficulty that arises from representing a directional tool transformation (e.g., Beisert, Massen, \& Prinz 2010; Müsseler \& Skottke, 2011; Takahashi, Diedrichsen, \& Watt, 2009; for reviews of tool use, see Heuer \& Sülzenbrück, 2013; Johnson-Frey, 2003).

This perspective raises the question of how tool-use actions can be interpreted from the perspective of ideomotor theory (Heuer \& Sülzenbrück, 2013; Kunde, Müsseler, \& Heuer, 2007; Ladwig, Sutter, \& Müsseler, 2012). Because a tool moves as a direct function of own actions, a tool is an action effect by definition. An important property of this effect, however, is that it is typically used as a means to cause further effects in the environment, which often renders a tool movement an intended (i.e., task-relevant) action effect. On a theoretical level, we therefore do not draw a strong distinction between the study of transformed and untransformed movements and the study of other task-relevant action effects that are either incompatible or compatible with the corresponding actions. This reasoning is further corroborated by empirical findings on the locus of either tool transformation effects or R-E compatibility effects in stages of information processing (Kunde, Pfister, \& Janczyk, 2012; Paelecke \& Kunde, 2007; Wirth et al., 2015). Using the psychological refractory period paradigm, these studies suggest that tool transformation effects and R-E compatibility effects share at least one common processing stage, which is typically interpreted as subserving response selection. These findings further suggest that representing a task-relevant action effect shares key features with representing a tool transformation.

\section{Experiment 3}

In Experiments 3 and 4, we aimed to complement the present approach to body-related action effects by exploring the temporal dynamics of body-related versus environment-related action effects. For environment-related effects, previous results have demonstrated crucial roles of effect anticipation during both response selection and response initiation (Kunde, 2003; Kunde, Koch, \& Hoffmann, 2004; Shin \& Proctor, 2012). More precisely, these studies assumed that anticipated effects can be activated with different strengths and that an action is executed eventually when this strength exceeds a certain threshold. Intentionally recollecting a possible effect, therefore, can be used to raise the subthreshold activation of an action relative to other actions (response selection), and it can be used to actually prompt the action (response initiation; Kunde et al., 2004; Shin \& Proctor, 2012).

To dissociate response selection processes from processes relating to response initiation, these studies employed a precue that informed about the to-be-performed action, but the action itself had to be initiated only after a variable CGI. Larger R-E compatibility effects were observed at short CGIs than at long ones (indicating a prominent role of effect anticipations in action selection, which is mainly relevant at short preparation intervals), whereas reliable R-E compatibility effects were still obtained at long CGIs, at which action selection had been completed (indicating a role of effect anticipations in action initiation, even after a proper response had already been selected).

In Experiment 3 we adopted this strategy, with the additional benefit of the present paradigm that it allows for studying not only the R-E compatibility effects for response selection and initiation, but also the influences of effect anticipations during response execution. In Experiment 3, we realized environment-related action effects by linking visual feedback to the slider movement, to replicate previous findings for such effects within the present setup. We expected to find reliable effects of R-E compatibility across all preparation intervals, but larger effects at short than at long intervals, evidenced by an interaction between R-E compatibility and CGI.

\section{Method}

Participants A new set of 24 participants was recruited (13 female, 11 male; four left-handed; mean age $=25.1$ years) and received monetary compensation. All participants reported normal vision and hearing and were naïve concerning the hypotheses of the experiment, and all provided written informed consent prior to the experiment. Two of the participants were excluded from all analyses due to high error rates $(>25 \%)^{3}$

Apparatus, stimuli, and procedure In Experiment 3, we used the same apparatus and stimuli as in Experiment 1. The trial procedure was slightly adjusted to allow for response preparation: A go signal was introduced (a short auditory signal played back via headphones) and was presented after

\footnotetext{
${ }^{3}$ Even when including all 24 participants in the analysis, the results for ITs and RTs did not change for the main effects of CGI, $F_{\mathrm{S}}>88.41, p \mathrm{~s}<$ .001 , and R-E mapping, $F \mathrm{~s}>24.69, p \mathrm{~s}<.001$, or for the interaction between CGI and R-E mapping, $F_{\mathrm{S}}>4.53, p \mathrm{~s}<.013$. No significant effects emerged for MTs, $F \mathrm{~s}>3.29$, $p \mathrm{~s}>.083$, however.
} 
target onset with a varying CGI (0 vs. 500 vs. 1000 vs. 2000 $\mathrm{ms}$ ). To provide visual feedback instead of tactile brush stimulation, the brush was constantly lifted during the experiment, and instead a little red dot was displayed at the center of the screen and moved vertically according to the slider: In compatible trials, both the slider and the red dot moved in the same direction (e.g., both the slider and the dot moved up); in incompatible trials, they moved in opposite directions (e.g., the slider moved up, the dot moved down). The red dot appeared simultaneously with the auditory go signal. After the target was displayed, participants had to withhold their response until the go signal was played back and the red dot appeared. The stimuli were identical to those of Experiment 1. The color of the targets informed participants of the location that the dot had to be moved to (up vs. down); the shape indicated the current compatibility relation between the slider and the dot (compatible vs. incompatible). The mapping of color to dot locations, as well as the mapping of shape and compatibility relation, was counterbalanced across participants. To accommodate the additional conditions, the block length was increased to 32 trials.

\section{Results}

From the experimental blocks, trials with premature responses (i.e., movements away from the center position during the dwell time) were discarded (3.1\%), with $3.7 \%$ in the R-E compatible conditions versus $2.4 \%$ in the R-E incompatible conditions, $t(21)=3.19, p=.004, d=0.54$. All errors were omitted $(2.3 \%$ of the trials), with $2.9 \%$ in the R-E incompatible conditions versus $1.8 \%$ in the R-E compatible conditions, $t(21)=2.55, p=.019, d=0.52$. Equally, all trials with initial movements in the wrong direction were excluded; they occurred on $4.4 \%$ of all trials, and equally often in both R-E compatibility conditions, $t(21)=1.73, p=.098, d=0.25$. Trials with ITs or MTs deviating more than 2.5 SDs from their cell mean were discarded as outliers, which were calculated separately for each participant, CGI, and compatibility condition $(3.9 \%)$, with $4.1 \%$ of trials being removed for the compatible R-E mapping, and $3.7 \%$ for the incompatible R-E mapping, $t(21)=1.87, p=.074, d=0.46$.

The three measures were then analyzed in separate $2 \times 4$ repeated measures ANOVAs with R-E Mapping (compatible vs. incompatible) and CGI (0/500/1000/2000 ms) as withinsubjects factors (see Fig. 6 for the corresponding means).

Initiation time First, we found a significant main effect of CGI, $F(3,19)=90.83, p<.001, \eta_{\mathrm{p}}{ }^{2}=.94$, with faster responses after longer response preparation. Responses were also faster in the compatible condition $(359 \mathrm{~ms})$ than in the incompatible condition $(445 \mathrm{~ms}), F(1,21)=30.60, p<.001$, $\eta_{\mathrm{p}}{ }^{2}=.59$. Crucially, the interaction between CGI and R-E mapping was significant, $F(3,19)=7.28, p=.002, \eta_{\mathrm{p}}{ }^{2}=$
.54 , with smaller R-E compatibility effects after longer CGIs (141 ms after the shortest CGI, $38 \mathrm{~ms}$ after the longest CGI), with sizeable R-E compatibility effects for each individual CGI, $t \mathrm{~s}>2.67, p \mathrm{~s}<.014$ (Fig. 6, left panel; note that a Bonferroni-corrected alpha level would correspond to $\alpha / 4=$ $.05 / 4=.0125$, with all effects being significant when assessed with the sequential Bonferroni-Holm procedure).

Movement time The main effect of R-E mapping was marginally significant, $F(1,21)=3.89, p=.062, \eta_{\mathrm{p}}{ }^{2}=.16$, with faster responses in the compatible condition (364 ms) than in the incompatible condition (389 ms). Both CGI and the interaction between CGI and R-E mapping failed to reach significance, $F \mathrm{~s}<1.24, p \mathrm{~s}>.323$ (Fig. 6, center panel).

Response time First, we observed a significant main effect of CGI, $F(3,19)=76.51, p<.001, \eta_{\mathrm{p}}{ }^{2}=.92$, with faster responses after longer response preparation. The main effect of R-E mapping was also significant, $F(1,21)=27.23, p<.001$, $\eta_{\mathrm{p}}{ }^{2}=.57$, with faster responses in the compatible condition (723 ms) than in the incompatible condition $(835 \mathrm{~ms})$. Crucially, the interaction between CGI and R-E compatibility again was significant, $F(3,19)=8.17, p=.001, \eta_{\mathrm{p}}{ }^{2}=.56$, with smaller R-E compatibility effects after longer CGIs (177 ms after the shortest CGI, $58 \mathrm{~ms}$ after the longest CGI) and a significant R-E compatibility effect for each individual CGI, $t \mathrm{~s}>2.65, p \mathrm{~s}<.015$ (Fig. 6, right panel).

\section{Discussion}

In Experiment 3, we tested for the temporal dynamics of anticipations relating to environmental effects. Overall, we replicated the results from previous studies by showing that R-E compatibility effects diminish with longer response preparation intervals, and "that the process influenced by the responses' effect occurs mainly in a relatively early phase of motor planning" (Shin \& Proctor, 2012, p. 369). Here, we have provided empirical evidence for this hypothesis by pinpointing that the decrease of the compatibility effect is mainly due to the planning and initiation of the response; the execution is mostly unaffected by the preparatory interval. Although we found evidence that both response selection (in terms of a stronger influence of R-E compatibility at short than at longer CGIs) and response initiation (in terms of a residual influence of R-E compatibility at even the longest CGI) are affected by effect anticipations, response execution barely produces a compatibility effect, and seems to be largely unaffected by effect anticipations.

The lack of an R-E compatibility effect on MTs seems to be at odds with studies in which researchers have observed a continued impact of anticipated action effects during the execution of mouse movements (Pfister, Janczyk, Wirth, et al., 2014; Wirth et al., 2015). Our result is especially surprising 


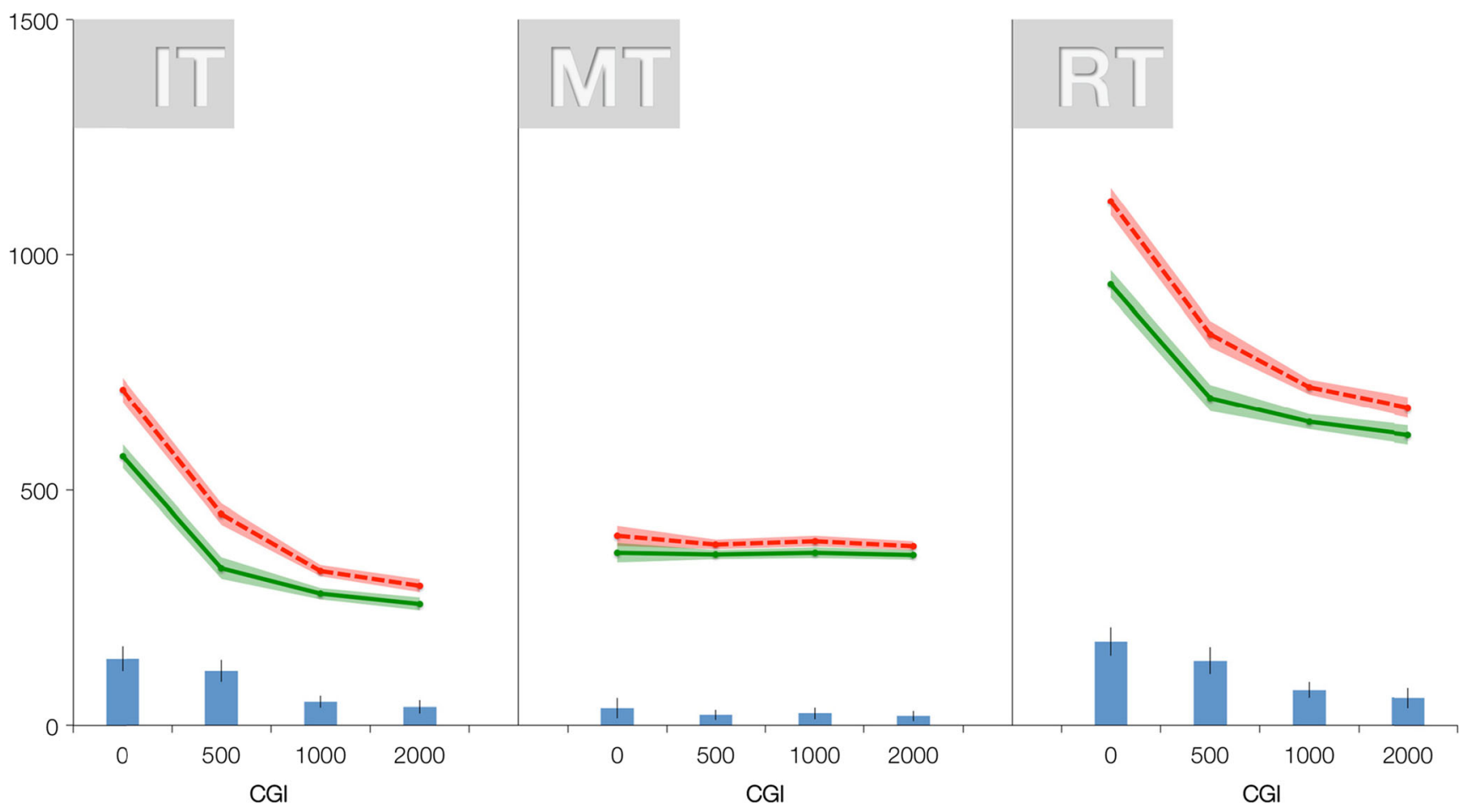

Fig. 6 Results of Experiment 3: Mean initiation times (IT, left panel), movement times (MT, center panel), and overall response times (RT, right panel), in milliseconds, shown separately for cue-go intervals (CGI; abscissa) and R-E mappings (continuous line = compatible; dashed line

$=$ incompatible). Each value is depicted with the corresponding standard error of the paired differences $\left(S E_{\mathrm{PD}}\right)$, calculated separately for each CGI (Pfister \& Janczyk, 2013). Bars indicate the sizes of the compatibility effects per CGI, with their $S E_{\mathrm{M}} \mathrm{S}$

given that the action effects of Experiment 3 (i.e., the dot movements caused by the slider) were highly task-relevant, since participants were instructed to move the dot to either the upper or the lower location. Please note, though, that the mean MTs in Experiment 3 were faster than the fastest MTs in Experiment 1, in which the R-E effect became very small as well. The very easy and consequently fast movements (all MTs $<400 \mathrm{~ms}$ ) might not allow for online monitoring of the participants' action effects (Elliott, Helsen, \& Chua, 2001; Spijkers \& Spellerberg, 1995). Moreover, the introduction of the go signal and the corresponding CGI might have prompted participants to spend more time on preplanning their response even at the short CGIs, so that execution of the highly prepared movements became more or less ballistic.

In any case, the time course of the R-E compatibility effects relating to response selection and initiation appears to be a robust result. Whether this pattern would also emerge for body-related action effects would be explored in Experiment 4.

\section{Experiment 4}

The critical question of Experiment 4 was whether the temporal dynamics that we found for environment- related action effects (Exp. 3) would also emerge for body-related feedback. We again employed the brush for tactile stimulation and allowed for response preparation by varying the levels of CGI. For body-related effects, three outcomes seem possible, considering that body-related sensations are likely to be tied more closely to body movements, while still being represented in a similar fashion: First, the impact of CGI might mirror the pattern of environment-related action effects, pointing toward a functional (and representational) equivalence of both types of effects. Second, R-E compatibility effects might decrease with increasing CGIs, but larger residual effects occur at long CGIs, pointing toward a more prominent role of body-related effects for movement initiation. Third, assuming that the role of body-related effects is confined to action initiation, one might even expect constant R-E compatibility effects across different CGIs.

\section{Method}

Participants A new set of 24 participants was recruited (21 female, three male; three left-handed; mean age $=27.8$ years ) and received monetary compensation. All participants reported normal vision and hearing and were naïve concerning the 
hypotheses of the experiment, and all provided written informed consent prior to the experiment. Three of the participants were excluded from all analyses due to high error rates $(>25 \%){ }^{4}$

Apparatus, stimuli, and procedure Experiment 4 was largely identical to Experiment 3 in terms of its apparatus, stimuli, and trial procedure. Instead of the visual feedback of a red moving dot on the screen, however, the brush inside the apparatus now provided tactile feedback on the participants' arms. In addition, the brush dropped simultaneously with the go signal, to provide an additional, tactile go signal. After the target had been displayed, participants had to withhold their response until the go signal was played back and the brush dropped. As in Experiment 1, the participants were instructed to move the brush toward the hand or crook position, according to the current compatibility relation between the slider and the brush.

\section{Results}

From the experimental blocks, trials with premature responses (i.e., movements away from the center position during the dwell time) were discarded (3.9\%), which occurred with a frequency of $4.6 \%$ in the R-E compatible conditions versus $3.2 \%$ in the R-E incompatible conditions, $t(20)=2.36, p=$ $.028, d=0.35$. All errors were omitted (3.4\% of the trials), which occurred equally often in the R-E compatible and incompatible conditions, $t(20)=0.31, p=.757, d=0.08$. Similarly, all trials with initial movements in the wrong direction were excluded; they occurred on $4.8 \%$ of all trials, equally often in each of the R-E compatibility conditions, $t(20)=1.17$, $p=.254, d=0.16$. Trials with ITs or MTs deviating more than $2.5 \mathrm{SD}$ s from their cell mean were discarded as outliers, which were calculated separately for each participant and compatibility condition (3.6\%) and occurred equally often in both R-E compatibility conditions, $t(20)=1.68, p=.107, d=0.27$.

The three measures were then analyzed in separate $2 \times 4$ repeated measures ANOVAs with R-E Mapping (compatible vs. incompatible) and CGI (0/500/1,000/2,000 ms) as withinsubjects factors (see Fig. 7 for the corresponding means).

Initiation time First, we observed a significant main effect of CGI, $F(3,18)=63.35, p<.001, \eta_{\mathrm{p}}{ }^{2}=.91$, with faster responses after longer response preparation. The main effect of

\footnotetext{
${ }^{4}$ Even when we included all 24 participants in the analysis, the results did not change for the main effects of CGI, $F_{\mathrm{S}}>27.39$, $p \mathrm{~s}<.001$, and R-E mapping, $F_{\mathrm{S}}>7.01, p \mathrm{~s}<.014$, for ITs and RTs. MTs returned the same, nonsignificant results for CGI and the interaction between CGI and R-E mapping, $F \mathrm{~s}<2.63, p \mathrm{~s}>.077$, while producing a significant main effect of R-E mapping, $F=19.38, p<.001$. The interaction between CGI and RE compatibility was significant for RTs, $F=3.88, p=.024$, but not for ITs, $F=1.31, p=.297$.
}

R-E mapping also reached significance, $F(1,20)=6.04, p=$ $.023, \eta_{\mathrm{p}}{ }^{2}=.23$, with faster responses in the compatible condition (429 ms) than in the incompatible condition (389 ms). Crucially, the interaction between CGI and R-E mapping was significant, $F(3,18)=3.67, p=.032, \eta_{\mathrm{p}}{ }^{2}=.38$, with smaller R-E compatibility effects after longer CGIs (109 ms after the shortest CGI, $35 \mathrm{~ms}$ after the longest), and a significant effect for the CGI of $0 \mathrm{~ms}, t(20)=3.04, p=.007, d=0.66$; a marginally significant effect for the CGI of $500 \mathrm{~ms}, t(20)=$ $2.05, p=.054, d=0.44$; and numerical effects for the longer CGIs, $t \mathrm{~s}>1.35, p \mathrm{~s}<.191$ (Fig. 7, left panel; with a significant effect only at the CGI of $0 \mathrm{~ms}$ when the effects were assessed with the sequential Bonferroni-Holm procedure).

Movement time The main effect of R-E mapping reached significance, $F(1,20)=18.45, p<.001, \eta_{\mathrm{p}}{ }^{2}=.48$, with faster responses in the compatible condition $(567 \mathrm{~ms})$ than in the incompatible condition $(601 \mathrm{~ms})$. No other effect was significant, $F \mathrm{~s}<1.55, p \mathrm{~s}>.237$ (Fig. 7, center panel).

Response time First, we observed a significant main effect of CGI, $F(3,18)=75.15, p<.001, \eta_{\mathrm{p}}{ }^{2}=.93$, with faster responses after longer response preparation. The main effect of R-E mapping was also significant, $F(1,20)=10.94, p=.004$, $\eta_{\mathrm{p}}^{2}=.35$, with faster responses in the compatible condition (996 ms) than in the incompatible condition $(1090 \mathrm{~ms})$. Crucially, the interaction between CGI and R-E compatibility was significant, $F(3,18)=5.13, p=.010, \eta_{\mathrm{p}}{ }^{2}=.46$, with smaller R-E compatibility effects after longer CGIs (162 ms after the shortest CGI, $59 \mathrm{~ms}$ after the longest CGI) with significant effects for all individual CGIs, $t \mathrm{~s}>2.48, p \mathrm{~s}<.022$ (Fig. 7, right panel; with all effects remaining significant when they were assessed via the Bonferroni-Holm procedure).

\section{Comparison to experiment 3}

To compare the sizes of the interactions of R-E compatibility with CGI in Experiments 3 and 4, we computed a betweenexperiments analysis. All three measures were analyzed in separate $2 \times 4 \times 2$ split-plot ANOVAs with R-E Mapping (compatible vs. incompatible) and CGI (0/500/1000/2000 $\mathrm{ms}$ ) as within-subjects factors and Experiment as a betweensubjects factor. To reduce redundancy, we focused on the interactions that included the factor Experiment, finding critical interactions for none of the three measures, $F_{\mathrm{s}}<1.26, p \mathrm{~s}>$ .302. This suggests that anticipations of both body-related and environment-related action effects follow the same temporal dynamics.

\section{Discussion}

With anticipation of the body-related action effects studied in Experiment 4, we found the same pattern of results as for 


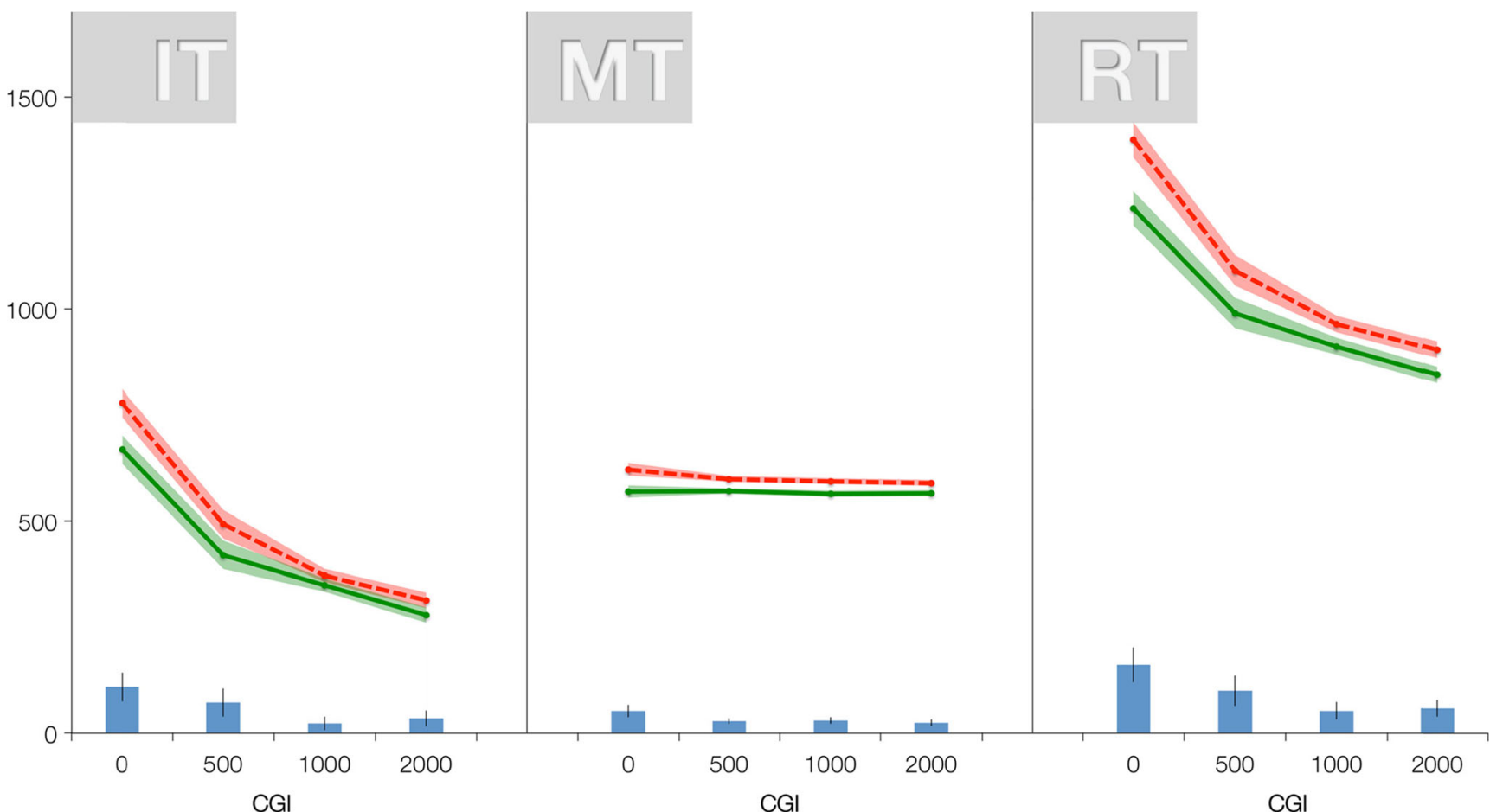

Fig. 7 Results of Experiment 4: Mean initiation times (IT, left panel), movement times (MT, center panel), and overall response times (RT, right panel), in milliseconds, shown separately for cue-go intervals (CGI; abscissa) and R-E mappings (continuous line = compatible; dashed line

$=$ incompatible). Each value is depicted with the corresponding standard error of the paired differences $\left(S E_{\mathrm{PD}}\right)$, calculated separately for each CGI (Pfister \& Janczyk, 2013). Bars indicate the sizes of the compatibility effects per CGI, with their $S E_{\mathrm{M}} \mathrm{S}$

anticipation of the environment-related effects of Experiment 3: Compatibility effects were reduced with long preparatory intervals, but still present at the longest preparatory interval of $2000 \mathrm{~ms}$. One apparent difference between the two experiments was that Experiment 4 produced a significant compatibility effect for movement times, whereas Experiment 3 did not. Additionally, the movements here were overall slower when participants controlled the brush (all $\mathrm{MTs}_{\mathrm{Exp} 4}>550$ $\mathrm{ms}$ ) than when they controlled the dot on the screen (all $\mathrm{MTs}_{\mathrm{Exp} 3}<400 \mathrm{~ms}$ ). Overall, slower movements render it easier to consider tactile feedback, which seemed to deteriorate performance in incompatible trials. Overall, the processes that advance the movement execution seem to be indifferent to whether the expected action effects are related to the agent's environment or the agent's body; both types of action effects seem to be recollected in early, response-planning stages. Consequently, we can assume that the anticipation processes for both effect types follow the same underlying temporal dynamics.

\section{General discussion}

In this series of experiments, we explored the anticipation of body-related action effects and their functions during action selection and initiation. A custom-made apparatus allowed us to manipulate how participants' hand movements of the

dominant hand were translated to a brush that stroked the forearm of the non-dominant hand. Such tactile feedback represents a type of body-related action effect that has been neglected by previous studies on effect-based action control (e.g., Ansorge, 2002; Chen \& Proctor, 2013; Gaschler \& Nattkemper, 2012; Hubbard, Gazzaley, \& Morsella, 2011; Kunde, 2001, 2003; Pfister et al., 2010; Pfister \& Kunde, 2013; Wirth et al., 2015; Yamaguchi \& Proctor, 2011).

In Experiment 1, we rendered the tactile action effects taskrelevant by instructing participants to move the brush toward either their hand or their arm crook while we manipulated the R-E compatibility relation between the slider and brush movements. Movements that produced spatially compatible tactile feedback were initiated and executed with more ease than were movements with incompatible tactile feedback. Thereby, we conceptually replicated the results of previous studies that had employed vibrotactile body-related feedback (Pfister, Janczyk, Gressmann, et al., 2014), but we found that our design produced considerably larger and more robust compatibility effects. Thus, the present use of continuous movements that were translated to continuous tactile feedback seemed to promote the inclusion of such tactile effects into action control, and the present data provide strong support for a role of body-related action effects in effect-based action control. Experiment 2a replicated the design of Experiment 1 , but we rendered the tactile feedback task-irrelevant by instructing participants to move the slider (rather than the 
brush) toward the hand or arm crook, irrespective of the ensuing tactile sensation. No R-E compatibility effects emerged in this experiment, indicating that tactile action effects only exert a strong influence when they are explicitly intended (as suggested by Exp. 1), but that they are easily ignored when this is not the case. The visual dominance of the hand movement was thought to overpower the tactile action effects here, but even when no visual feedback was provided (Exp. 2b), RE compatibility effects did not re-emerge, strengthening the idea that tactile action effects need to be a task-relevant feature to exercise influences on response selection and production. As we outlined above, these findings may alternatively be understood as a tool transformation that needed to be represented in Experiment 1 (because the brush movement was task-relevant in this case), whereas it was not necessary to represent the transformation in Experiments $2 \mathrm{a}$ and $2 \mathrm{~b}$ (because the brush movement was irrelevant to the task in these cases).

The pronounced role of task relevance also seems to correspond to settings in which the action effects are imagined rather than actually perceived (Pfister, Pfeuffer, \& Kunde, 2014). Here, R-E compatibility effects only emerged when participants had an explicit intention to anticipate (or imagine) the unperceivable effect. Both situations - the present experimental setup as well as the study of merely imagined action effectsdo not particularly encourage action coding in terms of those action effects that are manipulated experimentally. A stronger impact of task-irrelevant tactile action effects might therefore emerge for blocked rather than variable action-effect mappings, a possibility that remains to be studied in future experiments.

In Experiments 3 and 4, we further tested for the temporal dynamics of effect anticipations by adding a preparatory interval before the response. The R-E compatibility effects for both, environment-related feedback (Exp. 3) and body-related feedback (Exp. 4), diminished with longer preparation, indicating critical roles of both types of effects in action selection. Yet, significant R-E compatibility effects did emerge with preparation of up to $2000 \mathrm{~ms}$, showing a stable influence of $\mathrm{R}-\mathrm{E}$ compatibility even on response initiation. Response execution, however, was barely modulated by R-E compatibility, suggesting that the effect of anticipation processes takes place in the early stages of response planning and initiation (cf. Kunde, 2003; Kunde et al., 2004; Shin \& Proctor, 2012). Thus, anticipations of body-related action effects seem to follow a time course similar to that of anticipations of effects in the environment.

The present findings therefore support early formulations of ideomotor theory (e.g., Harleß, 1861; Herbart, 1825; James, 1890 ) in showing that body-related action effects can be used to access one's response repertoire. At the same time, the findings also highlight more recent theoretical positions (Hommel, 2009; Hommel, Müsseler, Aschersleben, \& Prinz, 2001) by showing that body-related effects are functionally identical to action effects in the environment. Moreover, body-related effects seem to be used mainly when they are a relevant feature of the current task (please note that the abovementioned interpretation in terms of tool transformations also applies to Exps. 3 and 4, since similar precueing effects have been reported with tool-use actions; Massen \& Prinz, 2007). This finding is in line with work on bimanual coordination (Kunde et al., 2009; Kunde \& Weigelt, 2005; Mechsner et al., 2001; Mechsner \& Knoblich, 2004) that has shown a stable advantage for coordinating actions that produced symmetrical visual feedback, irrespective of the symmetry of the body movements that had to be performed (for a critical discussion of the role of vision regarding the emergence of such symmetry effects, see Heed \& Röder, 2014).

Arguably, most actions include both body-related and environment-related action effects. When, for example, a young scientist is working on his next research article and pressing the keys on his keyboard, these actions produce both body-related effects that are accessible only to him (the proprioceptive feedback of his fingers exercising force, the tactile feedback on his skin) and environment-related action effects that can also be perceived by his colleague at the next desk (the letters appearing on the screen, the unbearable clicking noise of the keys). In this scenario, the visual and acoustic effects of the keypresses would be far more important and would easily dominate the body-related action effects when it comes to the typing movements of the young scientist. The suggested dominance of visual information during typing (in this case, the letters produced on the screen) is consistent with studies showing that even skilled typists have only limited explicit knowledge about their keypress actions, which seem to be exclusively represented in terms of the action effects caused on screen (Liu, Crump, \& Logan, 2010).

These possible differences seem to be a promising line for future research. In any case, however, it should be noted that the present investigation of tactile action effects - though it has yielded more promising and robust effects than previous attempts (Pfister, Janczyk, Gressmann, et al., 2014) - is still some way from the proprioceptive action effects that were central in early formulations of ideomotor theory (Harle $\beta$, 1861; James, 1890). More precisely, William James distinguished between "resident" and "remote" action effects:

If the ideas by which we discriminate between one movement and another, at the instant of deciding in our mind which one we shall perform, are always of sensorial origin, then the question arises, "Of which sensorial order need they be?" It will be remembered that we distinguished two orders of kinæsthetic impression, the remote ones, made by the movement on the eye or ear or distant skin, etc., and the resident ones, made on the moving parts themselves, muscles, joints, etc. (p. 518) 
In this terminology, the tactile effects employed in the present experiments would clearly be classified as "remote" ones. Whether or not true resident effects - that is, the proprioceptive signals from the moving limbs - come with special functional properties thus cannot be answered by the present results. Yet, the experiments presented here shed light on a type of action effect that had been captured mostly theoretically before, but that had not been put to a thorough empirical testing.

Author note This work was supported by the German Research Foundation (Grant No. KU 1964/9-1). We especially thank Georg Schüssler for constructing the apparatus that was used for this experimental series.

\section{References}

Adam, J. J., Nieuwenstein, J. H., Huys, R., Paas, F. G., Kingma, H., Willems, P., \& Werry, M. (2000). Control of rapid aimed hand movements: the one-target advantage. Journal of Experimental Psychology: Human Perception and Performance, 26, 295-312. doi:10.1037/0096-1523.26.2.295

Ansorge, U. (2002). Spatial intention-response compatibility. Acta Psychologica, 109, 285-299. doi:10.1016/S0001-6918(01)00062-2

Beisert, M., Massen, C., \& Prinz, W. (2010). Embodied rules in tool use: A tool-switching study. Journal of Experimental Psychology: Human Perception and Performance, 36, 359-372.

Blakemore, S.-J., Frith, C. D., \& Wolpert, D. M. (1999). Spatio-temporal prediction modulates the perception of self-produced stimuli. Journal of Cognitive Neuroscience, 11, 551-559. doi:10.1162/ 089892999563607

Blakemore, S.-J., Wolpert, D. M., \& Frith, C. D. (1998). Central cancellation of self-produced tickle sensation. Nature Neuroscience, 1, 635-640

Blakemore, S.-J., Wolpert, D., \& Frith, C. (2000). Why can't you tickle yourself? NeuroReport, 11, R11-R16.

Buetti, S., Juan, E., Rinck, M., \& Kerzel, D. (2012). Affective states leak into movement execution: Automatic avoidance of threatening stimuli in fear of spider is visible in reach trajectories. Cognition and Emotion, 26, 1176-1188. doi:10.1080/02699931.2011.640662

Chen, J., \& Proctor, R. W. (2013). Response-effect compatibility defines the natural scrolling direction. Human Factors, 55, 1112-1129.

Colavita, F. B. (1974). Human sensory dominance. Perception \& Psychophysics, 16, 409-412. doi:10.3758/BF03203962

Colavita, F. B., \& Weisberg, D. (1979). A further investigation of visual dominance. Perception \& Psychophysics, 25, 345-347.

Dale, R., \& Duran, N. D. (2011). The cognitive dynamics of negated sentence verification. Cognitive Science, 35, 983-996.

Elliott, D., Helsen, W. F., \& Chua, R. (2001). A century later: Woodworth's (1899) two-component model of goal-directed aiming. Psychological Bulletin, 127, 342-357. doi:10.1037/00332909.127.3.342

Elsner, B., \& Hommel, B. (2001). Effect anticipation and action control. Journal of Experimental Psychology: Human Perception and Performance, 27, 229-240. doi:10.1037/0096-1523.27.1.229

Freeman, J. B., \& Ambady, N. (2014). The dynamic interactive model of person construal: Coordinating sensory and social processes. In J. Sherman, B. Gawronski, \& Y. Trope (Eds.), Dual process theories of the social mind (pp. 235-248). New York, NY: Guilford.
Gaschler, R., \& Nattkemper, D. (2012). Instructed task demands and utilization of action effect anticipation. Frontiers in Psychology, 3, 578. doi:10.3389/fpsyg.2012.00578

Greenwald, A. G. (1970). Sensory feedback mechanisms in performance control: With special reference to the ideo-motor mechanism. Psychological Review, 77, 73-99. doi:10.1037/h0028689

Harleß, E. (1861). Der Apparat des Willens [The Apparatus of Will]. Zeitschrift für Philosophie und philosophische Kritik, 38, 50-73.

Heed, T., \& Röder, B. (2014). Motor coordination uses external spatial coordinates independent of developmental vision. Cognition, 132, 1-5. doi:10.1016/j.cognition.2014.03.005

Herbart, J. F. (1825). Psychologie als Wissenschaft, neu gegründet auf Erfahrung, Metaphysik, und Mathematik (Vol. 2). Königsberg, Germany: August Wilhelm Unzer.

Heuer, H., \& Sülzenbrück, S. (2013). Tool use in action: The mastery of complex visuomotor transformations. In W. Prinz, M. Beisert, \& A. Herwig (Eds.), Action science: Foundations of an emerging discipline (pp. 37-62). Cambridge, MA, USA: MIT Press.

Hoffmann, J., Lenhard, A., Sebald, A., \& Pfister, R. (2009). Movements or targets: What makes an action in action-effect learning? Quarterly Journal of Experimental Psychology, 62, 2433-2449.

Hommel, B. (1993). Inverting the Simon effect by intention: Determinants of direction and extent of effects of irrelevant spatial information. Psychological Research, 55, 270-279. doi:10.1007/ BF00419687

Hommel, B. (2009). Action control according to TEC (theory of event coding). Psychological Research, 73, 512-526. doi:10.1007/ s00426-009-0234-2

Hommel, B., Müsseler, J., Aschersleben, G., \& Prinz, W. (2001). The Theory of Event Coding (TEC): A framework for perception and action planning. Behavioral and Brain Sciences, 24, 849-878, disc. 878-937. doi:10.1017/S0140525X01000103

Hubbard, J., Gazzaley, A., \& Morsella, E. (2011). Traditional response interference effects from anticipated action outcomes: A responseeffect compatibility paradigm. Acta Psychologica, 138, 106-110. doi:10.1016/j.actpsy.2011.05.012

James, W. (1890). The principles of psychology (Vol. 1-2). Cambridge, MA: Harvard University Press.

Janczyk, M., Pfister, R., Crognale, M. A., \& Kunde, W. (2012). Effective rotations: Action effects determine the interplay of mental and manual rotations. Journal of Experimental Psychology: General, 141, 489-501. doi:10.1037/a0026997

Janczyk, M., Skirde, S., Weigelt, M., \& Kunde, W. (2009). Visual and tactile action effects determine bimanual coordination performance. Human Movement Science, 28, 437-449.

Janczyk, M., Yamaguchi, M., Proctor, R. W., \& Pfister, R. (2015). Response-effect compatibility with complex actions: The case of wheel rotations. Attention, Perception, \& Psychophysics, 77, 930940. doi:10.3758/s13414-014-0828-7

Johnson-Frey, S. H. (2003). What's so special about human tool use? Neuron, 39, 201-204.

Keller, P. E., \& Koch, I. (2006). Exogenous and endogenous response priming with auditory stimuli. Advances in Cognitive Psychology, 2, 269-276.

Keysers, C., Wicker, B., Gazzola, V., Anton, J. L., Fogassi, L., \& Gallese, V. (2004). A touching sight: SII/PV activation during the observation and experience of touch. Neuron, 42, 335-346.

Kunde, W. (2001). Response-effect compatibility in manual choice reaction tasks. Journal of Experimental Psychology: Human Perception and Performance, 27, 387-394. doi:10.1037/0096-1523.27.2.387

Kunde, W. (2003). Temporal response-effect compatibility. Psychological Research, 67, 153-159.

Kunde, W., Koch, I., \& Hoffmann, J. (2004). Anticipated action effects affect the selection, initiation, and execution of actions. Quarterly Journal of Experimental Psychology, 57A, 87-106. doi:10.1080/ 02724980343000143 
Kunde, W., Krauss, H., \& Weigelt, M. (2009). Goal congruency without stimulus congruency in bimanual coordination. Psychological Research, 73, 34-42.

Kunde, W., Lozo, L., \& Neumann, R. (2011). Effect-based control of facial expressions: Evidence from action-effect compatibility. Psychonomic Bulletin \& Review, 18, 820-826. doi:10.3758/ s13423-011-0093-x

Kunde, W., Müsseler, J., \& Heuer, H. (2007). Spatial compatibility effects with tool use. Human Factors, 49, 661-670.

Kunde, W., Pfister, R., \& Janczyk, M. (2012). The locus of tooltransformation costs. Journal of Experimental Psychology: Human Perception and Performance, 38, 703-714.

Kunde, W., \& Weigelt, M. (2005). Goal congruency in bimanual object manipulation. Journal of Experimental Psychology: Human Perception and Performance, 31, 145-156. doi:10.1037/00961523.31.1.145

Ladwig, S., Sutter, C., \& Müsseler, J. (2012). Crosstalk between proximal and distal action effects during tool use. Zeitschrift für Psychologie, $220,10-15$

Liu, X., Crump, M. J. C., \& Logan, G. D. (2010). Do you know where your fingers have been? Explicit knowledge of the spatial layout of the keyboard in skilled typists. Memory \& Cognition, 38, 474-484. doi:10.3758/MC.38.4.474

Massen, C., \& Prinz, W. (2007). Programming tool-use actions. Journal of Experimental Psychology: Human Perception and Performance, 33, 692-704.

Mechsner, F., Kerzel, D., Knoblich, G., \& Prinz, W. (2001). Perceptual basis of bimanual coordination. Nature, 414, 69-73.

Mechsner, F., \& Knoblich, G. (2004). Do muscles matter for coordinated action? Journal of Experimental Psychology: Human Perception and Performance, 30, 490-503. doi:10.1037/0096-1523.30.3.490

Müsseler, J., \& Skottke, E. M. (2011). Compatibility relationships with simple lever tools. Human Factors, 53, 383-390.

Paelecke, M., \& Kunde, W. (2007). Action-effect codes in and before the central bottleneck: Evidence from the psychological refractory period paradigm. Journal of Experimental Psychology: Human Perception and Performance, 33, 627-644. doi:10.1037/00961523.33.3.627

Pfister, R., \& Janczyk, M. (2012). Harleß’ Apparatus of Will: 150 years later. Psychological Research, 76, 561-565. doi:10.1007/s00426011-0362-3

Pfister, R., \& Janczyk, M. (2013). Confidence intervals for two sample means: Calculation, interpretation, and a few simple rules. Advances in Cognitive Psychology, 9, 74-80.

Pfister, R., Janczyk, M., Gressmann, M., Fournier, L. R., \& Kunde, W. (2014). Good vibrations? Vibrotactile self-stimulation reveals anticipation of body-related action effects in motor control. Experimental Brain Research, 232, 847-854. doi:10.1007/s00221-013-3796-6

Pfister, R., Janczyk, M., Wirth, R., Dignath, D., \& Kunde, W. (2014). Thinking with portals: Revisiting kinematic cues to intention. Cognition, 133, 464-473.

Pfister, R., Kiesel, A., \& Melcher, T. (2010). Adaptive control of ideomotor effect anticipations. Acta Psychologica, 135, 316-322. doi: 10.1016/j.actpsy.2010.08.006
Pfister, R., \& Kunde, W. (2013). Dissecting the response in responseeffect compatibility. Experimental Brain Research, 224, 647-655.

Pfister, R., Pfeuffer, C. U., \& Kunde, W. (2014). Perceiving by proxy: Effect-based action control with unperceivable effects. Cognition, 132, 251-261. doi:10.1016/j.cognition.2014.04.012

Pfister, R., Wirth, R., Schwarz, K. A., Steinhauser, M., \& Kunde, W. (2016). Burdens of non-conformity: Motor execution reveals cognitive conflict during deliberate rule violations. Cognition, 147, 93-99.

Posner, M. I., Nissen, M. J., \& Klein, R. M. (1976). Visual dominance: An information-processing account of its origins and significance. Psychological Review, 83, 157-171. doi:10.1037/0033-295X.83.2. 157

Rieger, M. (2007). Letters as visual action-effects in skilled typing. Acta Psychologica, 126, 138-153.

Shin, Y. K., \& Proctor, R. W. (2012). Testing boundary conditions of the ideomotor hypothesis using a delayed response task. Acta Psychologica, 141, 360-372. doi:10.1016/j.actpsy.2012.09.008

Song, J.-H., \& Nakayama, K. (2009). Hidden cognitive states revealed in choice reaching tasks. Trends in Cognitive Sciences, 13, 360-366. doi:10.1016/j.tics.2009.04.009

Spijkers, W., \& Spellerberg, S. (1995). On-line visual control of aiming movements? Acta Psychologica, 90, 333-348.

Spivey, M. J., Grosjean, M., \& Knoblich, G. (2005). Continuous attraction toward phonological competitors. Proceedings of the National Academy of Sciences, 102, 10393-10398. doi:10.1073/pnas. 0503903102

Stock, A., \& Stock, C. (2004). A short history of ideo-motor action. Psychological Research, 68, 176-188.

Takahashi, C., Diedrichsen, J., \& Watt, S. J. (2009). Integration of vision and haptics during tool use. Journal of Vision, 9(6), 3. doi:10.1167/ 9.6 .3

Washburn, M. F. (1908). The animal mind: A textbook of comparative psychology. New York, NY: Macmillan.

Weiskrantz, L., Elliott, J., \& Darlington, C. (1971). Preliminary observations on tickling oneself. Nature, 230, 598-599.

Wirth, R., Pfister, R., Foerster, A., Huestegge, L., \& Kunde, W. (in press). Pushing the rules: Effects and aftereffects of deliberate rule violations. Psychological Research. doi:10.1007/s00426-015-0690-9

Wirth, R., Pfister, R., Janczyk, M., \& Kunde, W. (2015). Through the portal: Effect anticipation in the central bottleneck. Acta Psychologica, 160, 141-151. doi:10.1016/j.actpsy.2015.07.007

Wirth, R., Pfister, R., \& Kunde, W. (2016). Asymmetric transfer effects between cognitive and affective task disturbances. Cognition and Emotion, 30, 399-416. doi:10.1080/02699931.2015.1009002

Wolfensteller, U., \& Ruge, H. (2011). On the timescale of stimulus-based action-effect learning. Quarterly Journal of Experimental Psychology, 64, 1273-1289.

Yamaguchi, M., \& Proctor, R. W. (2011). The Simon task with multicomponent responses: Two loci of response-effect compatibility. Psychological Research, 75, 214-226.

Zwosta, K., Ruge, H., \& Wolfensteller, U. (2013). No anticipation without intention: Response-effect compatibility in effect-based and stimulus-based actions. Acta Psychologica, 144, 628-634. doi:10. 1016/j.actpsy.2013.09.014 